\title{
Editorial
}

\section{Accessibility and Transportation Equity}

\author{
Anzhelika Antipova ${ }^{1, *}$, Salima Sultana ${ }^{2}$, , Yujie $\mathrm{Hu}^{3,4}{ }^{(\mathbb{D}}$ and James P. Rhudy Jr. ${ }^{5}$ \\ 1 Department of Earth Sciences, University of Memphis, Memphis, TN 38152, USA \\ 2 Department of Geography, Environment, and Sustainability, University of North Carolina-Greensboro, \\ Greensboro, NC 27412, USA; s_sultan@uncg.edu \\ 3 Department of Geography, University of Florida, Gainesville, FL 32611, USA; yujiehu@ufl.edu \\ 4 UF Informatics Institute, University of Florida, Gainesville, FL 32611, USA \\ 5 Department of Neurology, University of Tennessee Health Sciences Center, Memphis, TN 38163, USA; \\ jrhudy1@uthsc.edu \\ * Correspondence: antipova@memphis.edu
}

Received: 26 April 2020; Accepted: 28 April 2020; Published: 30 April 2020

\section{Introduction}

It is an honor to write an Editorial to this Special Issue (SI) of Sustainability. The SI addresses aspects of accessibility and equity and provides lessons from studies in various settings including the United States, China, Sweden, Poland, Peru, and Portugal to name a few, which collectively can contribute to a more sustainable and equitable transportation globally.

Accessibility is strongly tied to policymaking and thus has been extensively studied in a number of disciplines including transportation, geography, and urban planning. Accessibility can be defined in a variety of ways, recognizing influence by physical, political, economic, and social factors. It measures, for example, the potential of various opportunities for interaction, and the relative ease for people in an area to reach opportunities [1]. Various forms of accessibility are closely interdependent including transport availability and connectivity, communication, spatial, social, economic, physical, and temporal accessibility; thus, many novel measures are often taken to study different aspects and conceptualizations of accessibility. For example, as Mościcka et al. (2019) in this SI note, data from mobile phones can be used to study resident mobility, GPS-based location systems provide data on urban vehicle traffic, and the OpenStreetMap-based geospatial data are useful in research on urban public transportation networks, bicycle trails, as well as for studies on the availability of transportation for people with mobility restrictions. Additionally, Google Maps can provide an accurate measurement of travel times for different travel modes for various times of the day.

In this introduction to the Special Issue of Sustainability on accessibility and equity in transportation, we attempt to synthesize key lessons from the issue's fifteen substantive articles. These involve accessibility-related lessons including accessibility improvement in railways; optimizations of cross-border road accessibility, intercity networks, and pedestrian access to public transportation; as well as various aspects in urban transportation planning such as urban mobility, integration of bike-sharing, and electronically powered personal mobility vehicles. Other lessons cover equity-related aspects of transportation including the provision of the maximally full information to underserved populations to lessen the burden of unequitable access to urban facilities, ensuring socially equitable transportation planning and reducing burdens in commuting cost among low-income commuters. Finally, remaining lessons link equity back to accessibility with discussions on accessibility to public transport for disabled as well as visually impaired people, and equitable job access by poor commuters.

\section{Accessibility}

Researchers identify accessibility constraints and study how various urban form elements including location and design, and policy initiatives, can help achieve and/or improve accessibility. Accessibility 
analysis of a transportation system can identify gaps in accessibility and connectivity among regions in a whole country or indicate residential parts within a city underserved by public transport whose access could be improved. The results of the analysis might inform the future expansion of the transportation network.

In their study of accessibility, Fan et al. (2019) measure the spatial structure of a transportation network and strongly correlate accessibility with economic development. The socio-economic impacts of transportation development including those of railway networks in Europe have been well documented, while in the Chinese context, though recognizing that the improvement in accessibility drives regional and national population agglomeration, economic growth, and urbanization, yet the studies are lacking on the whole railway system. Fan and colleagues address this gap. In a detailed analysis, they investigated the spatial distribution of accessibility by measuring the various aspects of connectivity and accessibility of the Chinese railway network. The authors describe the improvement in accessibility of the Chinese railway network due to enhanced connectivity emerging over a seventy-year study period. The railway expansion of the seven regions has been analyzed and compared. Despite substantial improvement over 1949-2017, the spatial distribution pattern remains unbalanced: the underdeveloped west of China such as the northwest and southwest regions have the lowest accessibility, while places with higher economic level and population density have higher accessibility. Policy implications are to extend enhanced connectivity to the inland west, especially to poverty stricken countries, to support balanced regional growth and economic development.

In a similar study, Mościcka et al. (2019) add a comprehensive research on transport in Warsaw to the existing accessibility literature to better understand why passengers select a private car over buses or trams. The authors examine two integral components of accessibility: transport and land use, and the spatial distribution of people. Using Google Maps and a regular grid of points, travel times were measured for public transportation, car-based travel, walking and bicycling for various times of the day and various trip purposes including commuting to work, business trips, shopping, trips for health and educational services. They perform the spatial analysis and utilize a travel time perspective of transport accessibility to rank means of transportation in terms of efficiency for intraurban locations in a large European city. In the study set in Warsaw, Poland's city capital, car and bike transport are ranked higher than public transportation. In the context of city growth, effective and efficient public transportation might reduce travel by private cars in cities and decrease urban transport-related problems.). While bike commuting was found the second-most efficient way of transportation, authors acknowledge increasing popularity of electric scooters which seem to be most suited for very fast, short urban trips. The metro and railway system provide fast travel across long distances in a city for many passengers. The findings of this study may help improve the functioning of public transport.

Gao et al. (2019) note that taxi services can be a rich source of data on human travel activity, and that the Origin-Destination (O-D) pairs of urban taxi trips can provide researchers with information on human mobility. Non-negative mean factorization (NMF) has previously been used to assess human mobility, but it is hampered by its underlying assumptions. In this paper, Gao et al. add a novel spatiotemporal constraint (STC-NMF) to better account for temporal and spatial autocorrelation in taxi trips. They apply this new STC-NMF model to Beijing, using a pool of 15,033,149 O-D taxi trips to study mobility in the city. Using their novel method, they discovered four weekday arrival and departure patterns, and three weekend arrival and departure patterns within the city. Additional spatial interaction patterns were also found, with mixed-purpose trips being common during the weekdays and leisure trips being common during the weekends. This research demonstrated the potential strengths of the STC-NMF model, but more effort will be needed to improve it in the future.

This Special Issue also addresses another important accessibility issue on cross-border transport infrastructure. Accessibility in border regions is compounded by physical, political and infrastructural barriers and is generally uneven by regions. Christodoulou and Christidis take on this issue by measuring the accessibility and connectivity of border regions across the European Union using a combination of population density and the road network. This analysis is completed at a European wide scale, 
using population density measurements on a $1 \mathrm{~km} \times 1 \mathrm{~km}$ grid. Various accessibility indicators were calculated including a location indicator, a potential accessibility indicator and a network efficiency indicator. They found that there is a "clear border effect that affects accessibility in border regions" regardless of indicators one use. They found from the location indicator that the median travel time to reach activities in EU border regions was 49 minutes while potential accessibility identifies "large number" of cells with low potential accessibility, indicating that there are many isolated areas in border regions. Network efficiency was determined to be hindered likely by physical barriers along borders. The results of this research may be useful for informing policy decisions, and for identifying regions where equity and increased accessibility to opportunities needs to be improved.

Kaszczyszyn and Sypio-Dutkowska (2019) analyze accessibility to public transit stops for residents in Pomorzany, Szczecin, Poland. Generally, residents who access public transit opportunities walk to transit stops. Previous research has demonstrated that residents are willing to walk up to $400-580 \mathrm{~m}$ to reach public transit stops, but if this threshold is exceeded, their likelihood of walking to transit decreases by an order $50 \%$ for every additional $500 \mathrm{~m}$ that they have to walk. Two traditional methods of analyzing walking accessibility are the band method and the circular buffer method. In this research the authors attempt to (A) compare the performance of these two methods, (B) assess public transit accessibility in Pomorzany, and (C) delineate areas of the city with poor public transportation accessibility. Using the band method, the authors found that $46.4 \%$ of the walking network was "highly accessible" to public transit, while $13.5 \%$ of streets and paths were completely inaccessible. The authors also found that the band method was more accurate in its demarcation of accessibility compared to the circular buffer method. They also found that main streets in Pomorzany had higher accessibility to public transit than side streets, and that access along residential side roads needs to be improved.

Another important article examines the overlap (or lack thereof) between sustainable urban mobility policies and "healthy city" public health policies in practice by Louro et al. (2019). Using Lisbon, Portugal as a case study, the authors attempt to answer whether sustainable urban mobility policies "are contributing to healthy cities and, simultaneously, whether the domain of urban mobility is already a concern within public health policies". Using a content analysis methodology, the authors examined (A) planning instruments, and (B) the discourses and practices of municipal agents to see whether urban mobility issues and public health issues were a concern to each other's fields. They found that even though sustainable development and healthy cities are strongly linked, the two have weak domain presence in each other's fields. This contradiction may be caused by the different approaches of each movement. Whereas the public health "healthy city" movement is a bottom-up movement, urban sustainable mobility policies follow a top down model, especially in Europe. Despite the fact that various agents do not explicitly promote healthy city policies in their sustainable development schemes, these policies are still implicitly and informally carried out. The paper conclude that sustainable urban mobility policies are contributing to healthy cities, even if it is unintentional.

Another significant contribution made by Miao and Ni (2019) where they develop a new accessibility-based method to analyze intercity network vulnerability in the Yangtze River Delta of China. Building off of the 2015 work of Chen et al. [2], Miao and Ni refocus their Hansen-derived accessibility calculations around multimodal intercity connections. Using GDP as the main measure of a city's "attraction" along a network, Miao and Ni modeled the impacts that different node failures may have on the transportation network. They found that Nanjing, Shanghai, Suzhou and Hangzhou are the most important economic nodes in the Yangtze River Delta. Nanjing is the most vulnerable node of these four cities. This research demonstrated the applicability of a modified Hansen method in studying network vulnerability, and the associated accessibility impacts that may happen as a result.

Electrical Power-Assisted Mobility Vehicles (E-PMVs) are widely seen as an environmentally friendly transportation mode, especially for short trips. While E-PMVs do provide many benefits, they also have their share of potential drawbacks and implementation issues. Zagorkas and Burinskiene (2019) provides a systematic overview of literature on this topic to identify challenges for the implementation 
of E-PMVs, and brought attention to measures that may be needed for increasing the use of e-powered personal transports. They begin by describing the mechanical and functional properties of E-PMVs, highlighting their different designs and forms. While E-PMVs can take on many different shapes, their primary benefit is their power assistance, which allows them to cover bigger distances, ride at higher speeds, and cope with natural barriers like big inclines, windy, and hilly areas better than traditional bicycles. E-PMV use has exploded in many European countries in recent years, and while this growth has many positive aspects, it also has some issues. The growth of E-PMVs may impact existing transportation systems, with bicycles and walking suffering declines. Existing infrastructure designed for bicycles and walking may have to be re-designed to account for the higher speeds of E-PMVs, and additional safety measures may also need to be taken to keep pedestrians and riders alike safe from this new technology. Another issue of E-PMVs is environmental, with mass e-scooter graveyards being a serious problem in other parts of the world such as China. With these issues in mind, the authors suggest that E-PMVs should be regulated and restricted by European governments and that private users should be fined for improper parking and riding behavior.

Environmentally friendly and beneficial to public health transportation such as cycling contribute to sustainable urban development. Bike-sharing systems where individuals use a bicycle on an 'as-needed' basis but avoid the costs and responsibilities related to bicycle ownership date back to the mid-1960s Europe. Since then, bike-sharing systems became popular in China. Two important and different stages for their development include station-based and station-less bike-sharing systems which appeared in China successively. Wang et al. (2019) reviewed the emergence, growth, and consolidation of station-less bike-sharing systems in Chinese cities. To examine the attitudes of, and usage by, different categories of user and the attitudes of non-users, the authors conducted a survey which was distributed via the social media portal 'WeChat'. The findings are that this service developed unevenly beginning with urban regions to the east and southeast of China. Other modes of transport. Policy implications are that urban bike-sharing services should be integrated with urban planning of such issues as road usage and traffic policing.

\section{Equity}

Ensuring accessible and equitable transportation is an ongoing challenge. Using the 2012-2016 Census Transportation Planning Products (CTPP), Anzhelika Antipova (2020) measured the journey-to-work patterns-specifically, commuting distance-of low-income workers in Memphis metropolitan area, TN that has been undergoing decreased economic opportunity. The analysis found that commuters living in low-income neighborhoods, on average, had significantly shorter commute distance $(7$ miles) than the general commuters (10 miles) in the region. This pattern is consistent with existing studies examining other urban areas such as Baton Rouge, LA [3,4]. Different from these existing studies, the author then took a step further by looking at more localized pattern given that such a global finding may not be observed in every low-income neighborhood. Take long commutes (over 50 miles) as an example, it was reported that a significantly greater proportion of workers from low-income neighborhoods traveled longer than workers from high-income neighborhoods. The findings thus revealed unequal spatial access to jobs, measured by distance to jobs, between low-income and high-income neighborhoods. Policy implications are that the planning authority may adopt an income-based policy to target specifically at those low-income neighborhoods that suffer from long commute.

Yet another important topic of research inquiry and of this Special Issue is inspired by the social justice theory and investigates equity in access to resources or public services between the haves and have nots. Kim and Wang (2019) modelled the availability of public daycare and kindergarten in countries with decreasing fertility rates. Place accessibility is measured in order to identify high- and low access areas (e.g., underserved areas in terms of healthy foods such as food deserts, healthcare services, jobs, etc.) using different methods that might contribute to future policies on the spatial planning of childcare facilities and a development of measures for improving overall accessibility of 
these facilities, especially critical for underserved and socially disadvantaged population segments. Studies of spatial accessibility to childcare facilities have grown since the 2000s due to their recognized importance in children's health, safety, and skill development on one hand, and on the other hand, because insufficient public services, including childcare, have been a chronic problem in a modern metropolis. Their study uses the GIS-based two-step floating catchment area model to analyze an existing geographic disparity in the public daycare and kindergarten accessibility across Seoul, the capital city of South Korea. The highest priority for the municipal government should be placed on areas burdened with a shortage of childcare facilities and low socio-economic status. In the study, the "Double-Disadvantage Regions" have been identified where the provision of childcare there is desirable for promoting social equity. The results inform parents of neighborhoods with adequate access in Seoul, South Korea and suggest family policy focused on improving accessibility of childcare. Policy implications are that parents with superior information about the likely availability of these services will make better decisions about where to raise families, especially among underserved populations.

Rye and Wretstrand (2019) investigated social equity in transportation planning using a theoretical framework developed by Geurs et al. [5]. Specifically, they examined national long-term transportation planning policies in two European countries-Sweden and Scotland-in terms of the associated social impacts. Despite the two countries having different definitions of social impacts, they share a consistent goal of improving social equity in making national transportation plans. However, evidence from looking at national measures and spending revealed that the goal was not achieved in both countries. For instance, compared to bus project, large-scale rail and road projects received disproportionally greater budget allocation, thus creating a less equitable transportation system. Furthermore, such inequality is projected to be increasing.

\section{Equity Linked Back to Accessibility}

Various indicators measure different accessibility conceptualizations and connectivity and may provide complementary information about the accessibility of areas of the whole country such as Fan et al.'s (2019) study in this special issue. Vanessa Stjernborg (2019) also has conducted a qualitative content analysis to understand what barriers are experienced by travelers on public transport and why they experience them as barriers. Stjernborg brings us back full circle to equity linked with accessibility. This manuscript demonstrates improvement opportunities as evidenced by qualitative content analysis of feedback from disabled people in Sweden regarding accessibility to public transport. Policy implications include less emphasis on timetables and more emphasis on flexibility and sensitivity toward the disabled.

Oviedo et al. (2019) examine the accomplishment of Bus Rapid Transit (BRT) project in connecting resident workers to jobs in Lima, Peru with a particular focus on low-income neighborhoods in the peripheries of the city. The overall goal of this study is to evaluate whether this project has led to improved job accessibility for low-income workers. To do so, the authors compared the accessibility to employment before (2004) and after (2012) the construction of the BRT project. They found that the BRT project reduced travel times for the general commuters. The benefits vary significantly across neighborhoods, however. Specifically, affluent neighborhoods were reported to enjoy improved job access because of this project, whereas neighborhoods with high concentrations of poor populations experienced significantly decreased accessibility to jobs after the completion of the BRT system. Such a variation revealed an equity gap in the role of the BRT system in improving commuters' access to jobs in Lima. To achieve a system of more equitable access, the study further suggested several policy interventions including targeted fare subsidies for low-income groups, fare integration with other forms of public transportation, and future expansion of the BRT in identified vulnerable neighborhoods.

Liu et al. (2019) carried out a study evaluating the performance of current transportation system in Tianjin, China in supporting the visually impaired people accessing their daily activity locations such as disabled service centers and parks. They first conducted a survey examining the mode choice of this particular population group. Results indicated that these people when traveling alone mainly 
travel on foot but not use public transportation. Hence, they then focused on assessing to what extent the existing blind sidewalk system provide these people access to their daily activity destinations by comparing distributions of blind sidewalks and these road users' activity space. Results suggested that the layout of current blind sidewalks fall short of meeting the travel needs of this group of people, indicating inequality in accessibility of transportation system in Tianjin, China. Besides, this study provided guidance and suggestions on future upgrade of transportation system in the city for addressing the inequality issue. Findings and policy implications would be helpful for planners and policymakers aiming to improve spatial accessibility to daily activities for visually impaired people and other disabled groups.

\section{Conclusions}

This Special Issue is dedicated to studying accessibility and transportation equity. These included studies found significant inequality in transportation accessibility, regardless of region or how it is defined. For example, Antipova (2020) found that some low-income neighborhoods suffered from significantly long commute cost—-measured by mileage—relative to the general residents in Memphis Metropolitan Area, TN. Oviedo et al. (2019) concluded that the completion of BRT had led to a significant decline in accessibility to employment in socioeconomically disadvantaged neighborhoods, whereas it led to an improvement in affluent neighborhoods in Lima, Peru.. Liu et al. (2019) reported that the current layout of the blind sidewalk system significantly underserved visually impaired people in Tianjin, China. At a national scale, Rye and Wretstrand (2019) found disproportionally greater spending on large-scale rail and road projects than bus projects, despite both countries clearly stating social equity in national transportation planning in Sweden and Scotland. These evidences imply that significant inequality still exists in current transportation systems and future planning around the world. This demonstrates an imminent need for more targeted and effective planning strategies as detailed in these papers.

Acknowledgments: We thank all contributors of this Special Issue as well as the editorial staff of MDPI Sustainability Journal to make this Special Issue a success.

Conflicts of Interest: The authors declare no conflict of interest.

\section{References}

1. Hu, Y.; Downs, J. Measuring and visualizing place-based space-time job accessibility. J. Transp. Geog. 2019, 74, 278-288. [CrossRef]

2. Chen, X.-Z.; Lu, Q.-C.; Peng, Z.-R.; Ash, J.E. Analysis of Transportation Network Vulnerability under Flooding Disasters. Transp. Res. Rec. J. Transp. Res. Board 2015, 2532, 37-44. [CrossRef]

3. Hu, Y.; Wang, F.; Wilmot, C.G. Commuting variability by wage groups in Baton Rouge, 1990-2010. Appl. Geog. 2017, 3, 14-29. [CrossRef]

4. Hu, Y.; Wang, F. GIS-Based Simulation and Analysis of Intra-Urban Commuting; CRC Press: Boca Raton, FL, USA, 2018.

5. Geurs, K.T.; Boon, W.; Van Wee, B. Social impacts of transport: Literature review and the state of the practice of transport appraisal in the Netherlands and the United Kingdom. Transp. Rev. 2009, 29, 69-90. [CrossRef] 